\title{
Exfoliative esophagitis: endoscopic and clinical profile of patient with severe chronic malnutrition
}

\author{
Andrey P. Kiryukhin ${ }^{1}$, Pavel V. Pavlov ${ }^{1}$, Alexander S. Tertychnyy ${ }^{2}$, Kirill B. Puzakov ${ }^{3}$, and \\ Ramin T. Rzayev ${ }^{3}$
}

${ }^{1}$ Endoscopy Unit, ${ }^{2}$ Department of Pathology, ${ }^{3}$ Department of Radiology, The Second University Clinic, I.M. Sechenov First Moscow State Medical University (Sechenov University), Moscow, Russia

Received: June 10, 2020

Revised : June 17, 2020

Accepted: July 2, 2020

\section{Correspondence to}

Andrey P. Kiryukhin, M.D.

Tel: +7-9035066153

Fax: +7-4992483432

E-mail: a.p.kiryukhin@gmail.com https://orcid.org/0000-0001$5685-8784$
A 32-year-old woman was admitted to the hospital with recurrent moderate pain in the epigastrium and the right side after eating, along with constipation, periodic diarrhea, and eructation. The patient had a medical history of irritable bowel syndrome with mixed bowel habits; she had limited food intake because of abdominal pain in the previous 2 years. The patient had no unhealthy habits (no alcohol, tobacco, oral, or intravenous drug abuse), her medication intake history excluded sunitinib and dabigatran; and she had no autoimmune disorders. Her physical examination revealed a low body mass index $\left(15.7 \mathrm{~kg} / \mathrm{m}^{2}\right)$. No rash, hematomas, or petechiae were present on the skin. Laboratory examinations, abdominal ultrasonography, and colonoscopy with histopathological ex-
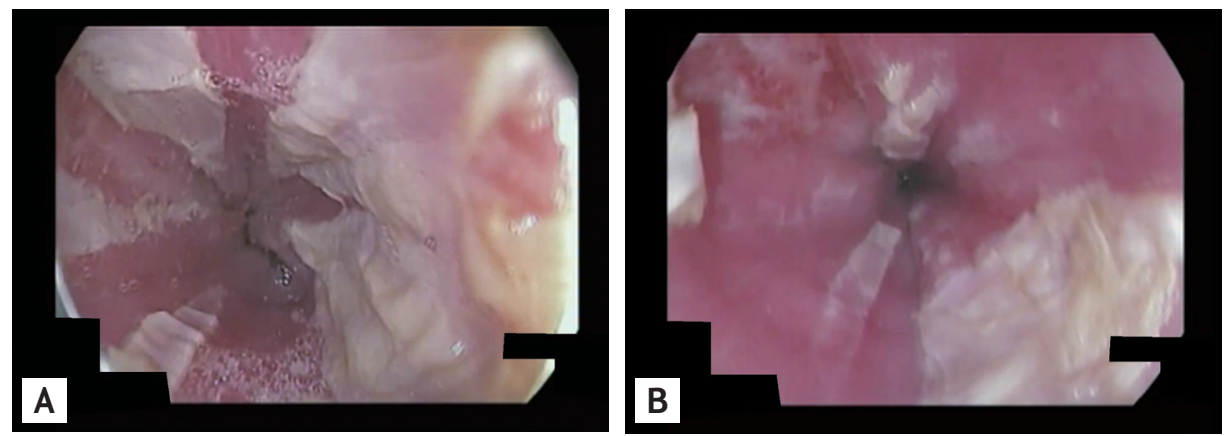

Figure 1. (A) Upper gastrointestinal (GI) endoscopy showing longitudinal sloughing and detachment of mucosa in the lower thoracic esophagus. (B) Upper GI endoscopy showing longitudinal sloughing and detachment of mucosa in the lower thoracic esophagus with mild redness after separation of mucosa by a distal end portion of an endoscope. 


\section{KJIM}

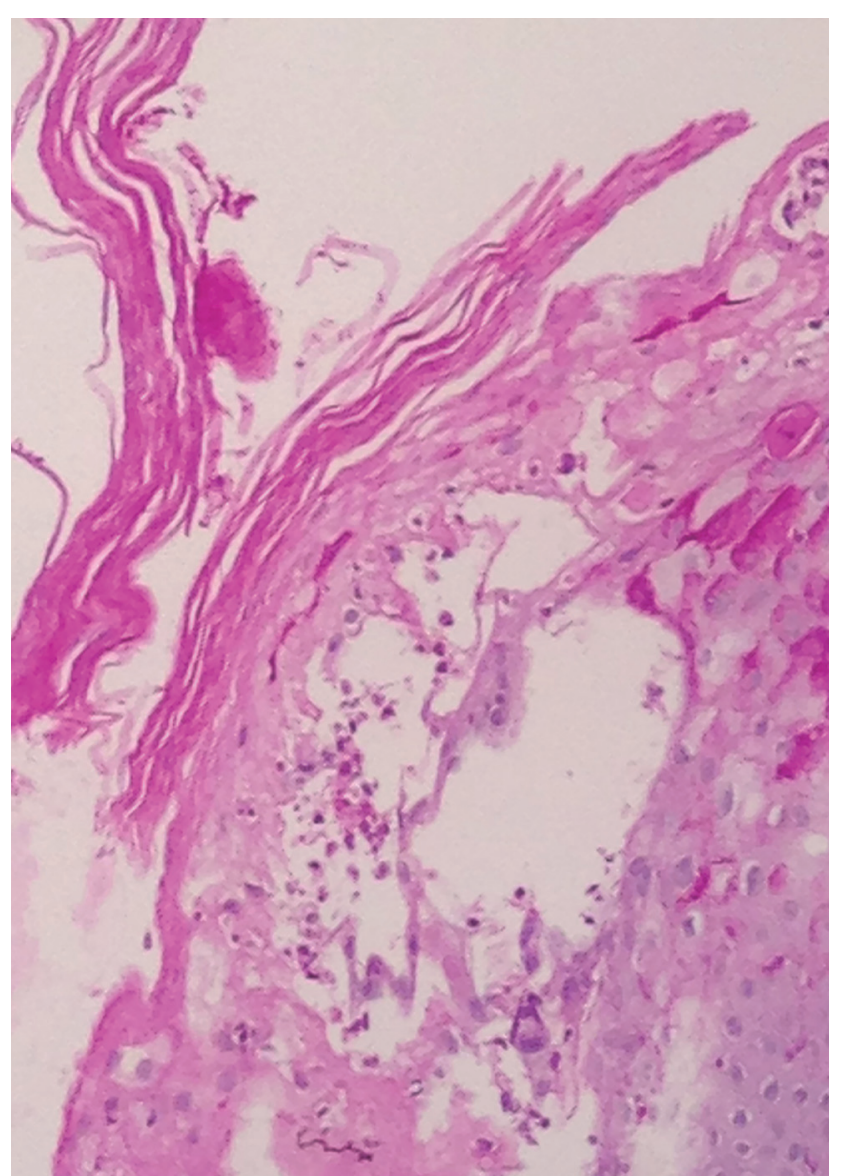

Figure 2. Histopathological examination of the biopsy specimen Histopathological section demonstrated an esophageal squamous epithelium dissected in upper layers with parakeratosis, cavity formation, and slight infiltration of leukocytes. Staining of the specimen for fungal colonies yielded negative results (periodic acid-Schiff stain, $\times 150$ ). gitis was confirmed. She was treated by drip infusion of a proton pump inhibitor, enteral nutrition, and metabolic supplements, and positive effects were achieved.

Exfoliative esophagitis is a desquamative esophageal disorder, involving sloughing of the superficial mucosa; the reported incidence is $0.03 \%$. The cause is poorly understood; the condition can be idiopathic or induced by drugs, food, or autoimmune disease. It is usually asymptomatic but can be associated with epigastric pain, nausea, and odynophagia.

We received informed consent from the patient for the publication of this case.

\section{Conflict of interest}

No potential conflict of interest relevant to this article was reported. 